\title{
Numerical Investigation of an Axisymmetric Model Scramjet Assisted with Cavity of Different Aft Wall Angles
}

\author{
Guangwei Ma $\mathbb{D}$, Mingbo Sun $\mathbb{D}^{\mathbb{D}}$, Guoyan Zhao, Pei Liu, Tao Tang, Li Fan, and Hongbo Wang
}

Science and Technology on Scramjet Laboratory, National University of Defense Technology, Changsha, Hunan 410073, China

Correspondence should be addressed to Mingbo Sun; sunmingbo@nudt.edu.cn

Received 9 June 2021; Accepted 6 July 2021; Published 3 August 2021

Academic Editor: Linda L. Vahala

Copyright (C) 2021 Guangwei Ma et al. This is an open access article distributed under the Creative Commons Attribution License, which permits unrestricted use, distribution, and reproduction in any medium, provided the original work is properly cited.

\begin{abstract}
An axisymmetric model scramjet assisted with cavity flameholder is numerically investigated. Three-dimensional Reynoldsaveraged Navier-Stokes simulation is carried out to reveal the fuel mixing and combustion characteristics. The simulation results show reasonable agreements with experimental data. The analysis indicates that the axisymmetric and rectangular scramjet has some similarities to the cavity shear layer in the nonreacting flow field. The configuration of the cavity shear layer changes hugely due to the significant chemical reaction and heat release in the reacting flow field. Typically, two more configurations with different cavity aft wall angles are compared with the experimental configuration to optimize the configuration of the cavity. When the cavity aft wall angle is small, the cavity shear layer bends to the cavity floor and more fuel enters into and stays in the cavity, which results in poor fuel mixing performance. With the increase of the aft wall angle, the fuel distributes more uniformly and the fuel mixing efficiency improves. In the reacting flow field, the volume of the cavity full of hot products and free radicals increases while the interaction between the cavity and main flow decreases with the increase of the aft wall angle. The improved combustion efficiency shows that larger cavity volume weighs more than reduced interaction between the cavity and main flow. The combustion is more violent in the case with a larger aft wall angle. Therefore, a proper increase of the aft wall angle is beneficial to the performance of cavity-assisted axisymmetric scramjet when designing the cavity flameholder.
\end{abstract}

\section{Introduction}

Hypersonic vehicles have the ability to fly at Mach 5 in nearspace. The air-breathing scramjet engine, which is the main part of the propulsion system of hypersonic vehicles, has drawn wide attention because of its excellent performance [1]. The researches on scramjet with rectangular crosssection have provided detailed and useful information for the development of hypersonic vehicles [2]. However, Bulman and Siebenhaar [3] emphasized the advantages of circular or axisymmetric cross-section scramjet (expressed as "axisymmetric scramjet" for simplicity in the rest of this article), such as good strength, light weight, small friction, and total pressure loss. The axisymmetric scramjet has been promoted to become an international consensus.

With the development of the experimental and numerical technology [4], some fundamental studies of the axisymmetric cross-section scramjet were carried out, and the mechanism of the flow and combustion in the scramjet was gradually revealed. Peterson et al. [5] numerically investigated the fuel distribution in an axisymmetric inlet-fueled scramjet and found that some extra and beneficial vortices formed and improved the fuel mixing efficiency remarkably. Landsberg et al. [6] improved the performance of a rectangular-toelliptical shape-transitioning scramjet. Employing a special "manipulator jet," the vortices inside the circular scramjet were changed and the fuel mixing and combustion efficiency improved, which implied that the length of the scramjet could be reduced. The experimental research of axisymmetric scramjet has also made some progress. Liu et al. [7] experimentally investigated the effect of single or dual jet injection in an axisymmetric scramjet with the variation of the injector Mach number. They found that the shape of the flame showed similarity when the injector Mach number changed. The 
penetration depth of the parallel dual jet increased because of the blockage effect of the first jet, and the distance between two jets should be optimized.

Due to the complexity of applying the flameholder in the axisymmetric scramjet, most of the studies focused on the field of high flight Mach number. Under this special condition, the flameholder could be removed. However, the flameholder, such as cavity [8] and strut [9], is essential under Mach 5 flight condition. The cavity flameholder [10], which evolves from the backward-facing step [11], has advantages over other flameholding schemes due to its low resistance and structural simplicity. The characteristics of the cavity have been extensively studied for the rectangular cross-section scramjet [12]. Although there are many difficult problems with the study of the cavity-assisted axisymmetric scramjet, there are still several studies in recent years. Vanyai et al. [13] carried out an experiment on the axisymmetric combustor assisted with the cavity at flight condition of Mach 7-8 and found that the flame structure became pretty different with the variation of the equivalence ratio. An axisymmetric scramjet with a cavity was experimentally and numerically studied by Denman et al. [14]. The experiment showed that the ethylene and hydrogen could be ignited and achieve stable combustion, while methane led to the extinguishment of the flame. Liu et al. [15] carried out experiments on the scramjet with an axisymmetric cavity at Mach 4.5. The experimental results showed that the existence of a cavity could promote the growth of a flame structure and enhance fuel mixing. Compared with the configuration without a cavity, the wall pressure increased significantly in the cavity-assisted scramjet.

In order to optimize the performance of the scramjet, various parameters, including cavity length-to-depth ratio [16], injection scheme [17], cavity position [18], and cavity aft wall angle [19], have been studied. However, most of the studies about the parameters are based on the rectangular scramjet due to the tricky problem existing in the axisymmetric scramjet. Only a few studies investigate the effect of the cavity parameters on the axisymmetric scramjet. Jeyakumar et al. [20] carried out a series of experiments to reveal the supersonic flow characteristics of an axisymmetric cavity without fuel injection. The effect of the aft wall angle was studied, and they found that the cavity drag could be reduced with the decreasing of the aft wall angle. Yao et al. [21] numerically investigated the differences between the elliptic and axisymmetric scramjet combustors and found that the special convex elliptic section played the role of a large open cavity, which would improve the fuel combustion efficiency. Liu et al. [22] experimentally investigated a typical model axisymmetric scramjet with the variation of wall divergent angle through experiments. The study showed that there existed a state where the heat release and the wall divergent angle reach the balance, and the flame could be quenched when the wall divergent angle became too large.

Although the investigation of the axisymmetric scramjet assisted with a cavity has been launched gradually, the detailed flow characteristics have not yet been explained clearly. The effect of the parameters, especially the cavity aft wall angle, on the flow field is very essential to determine a suitable cavity. A detailed study of the characteristics of the flow field and the effect of aft wall angle will lead to a more thorough understanding of the axisymmetric scramjet assisted with cavity and then provide some references for the engineering application. In this article, a model axisymmetric scramjet assisted with cavity is numerically investigated and the cavity aft wall angle is optimized to find a better configuration. Threedimensional compressible flamelet-based Reynolds-averaged Navier-Stokes (RANS) simulation is employed to show the details of the flow fields with or without combustion. The corresponding experiments carried out by Liu et al. [23] are compared to show the reliability of the simulation. The simulation is basically consistent with the experiment, and then, the analysis of the flow field is conducted, and the effect of the cavity aft wall angle is investigated.

\section{Description of Numerical Approach}

The in-house code in this article is a three-dimensional flamelet-based RANS simulation solver. The code is designed to solve the internal supersonic combustion problem in the scramjet, which contains complex supersonic turbulent reacting flow. The code has been verified in previous studies [24] for the supersonic mixing process. In this article, the flamelet/progress variable (FPV) combustion model is implanted into the code to simulate the supersonic combustion process, and the following analysis will prove that the code can solve the problem properly. Although the RANS simulation omits some details of the flow field, the important characteristics can still be captured. In this article, it is the overall performance that we should focus on, and the resource for the RANS simulation is pretty acceptable for parameter study. For the present study, the results can provide enough information, and the previous study [25] has proved that the flamelet-based RANS simulation is capable of calculating the supersonic combustion flow field. Therefore, the flamelet-based RANS simulation is applied in the current study.

2.1. Governing Equations for Flamelet-Based RANS Simulation. For supersonic turbulent combustion, the governing equations for flamelet-based RANS simulation can be written as follows:

$$
\begin{gathered}
\frac{\partial \bar{\rho}}{\partial t}+\frac{\partial \bar{\rho} \tilde{u}_{i} \tilde{u}_{j}}{\partial x_{j}}=0, \\
\frac{\partial \bar{\rho} \tilde{u}_{i}}{\partial t}+\frac{\partial \bar{\rho} \tilde{u}_{i} \tilde{u}_{j}}{\partial x_{j}}=-\frac{\partial \bar{p}}{\partial x_{j}}+\frac{\partial \bar{\tau}_{i j}}{\partial x_{j}}+\frac{\partial \bar{\tau}_{i j}^{R}}{\partial x_{j}}, \\
\frac{\partial \bar{\rho} \tilde{E}}{\partial t}+\frac{\partial \bar{\rho} \tilde{E} \tilde{u}_{j}}{\partial x_{j}}=-\frac{\partial \bar{p} \tilde{u}_{j}}{\partial x_{j}}+\frac{\partial}{\partial x_{j}}\left(\frac{\mu_{t}}{\operatorname{Pr}_{t}} \frac{\partial \tilde{h}}{\partial x_{j}}\right)-\frac{\partial \bar{q}_{j}}{\partial x_{j}}+\frac{\partial \tilde{u}_{i} \bar{\tau}_{i j}}{\partial x_{j}}+\frac{\partial \tilde{u}_{i} \bar{\tau}_{i j}^{R}}{\partial x_{j}}, \\
\frac{\partial \bar{\rho} \tilde{Z}}{\partial t}+\frac{\partial \bar{\rho} \tilde{Z} \tilde{u}_{j}}{\partial x_{j}}=\frac{\partial}{\partial x_{j}}\left[\left(\bar{\rho} D_{Z}+\frac{\mu_{t}}{\mathrm{Sc}_{Z}}\right) \frac{\partial \tilde{Z}}{\partial x_{j}}\right], \\
\frac{\partial \bar{\rho} \tilde{C}}{\partial t}+\frac{\partial \bar{\rho} \tilde{C} \tilde{u}_{j}}{\partial x_{j}}=\frac{\partial}{\partial x_{j}}\left[\left(\bar{\rho} D_{C}+\frac{\mu_{t}}{\mathrm{Sc}_{C}}\right) \frac{\partial \tilde{C}}{\partial x_{j}}\right]+\overline{\dot{\omega}}_{C},
\end{gathered}
$$


where the averaged heat flux vector is as follows:

$$
\bar{q}_{j}=-\left(\lambda+c_{p} \frac{\mu_{t}}{\operatorname{Pr}_{t}}\right) \frac{\partial \tilde{T}}{\partial x_{j}}-\sum_{s=1}^{N} \frac{\mu}{\mathrm{Sc}} \frac{\partial \tilde{Y}_{s}}{\partial x_{j}} \tilde{h}_{s} .
$$

In order to simplify the complex calculation, the following algebraic model for the variance of the mixture fraction is applied in our code, in which $\tilde{\varepsilon}$ is the turbulent dissipation rate:

$$
\widetilde{Z^{\prime \prime 2}}=\frac{C_{z} \mu_{t} \nabla \tilde{Z} \cdot \nabla \tilde{Z}}{\bar{\rho} \tilde{\varepsilon} S c_{t}}
$$

In the above equations, $\bar{\rho}, \tilde{u}_{i}, \tilde{E}, \tilde{Z}, \tilde{C}$, and $\widetilde{Z^{\prime \prime 2}}$ are the averaged density, velocity, total energy, mixture fraction, progress variable, and variance of the mixture fraction. $c_{p}, \lambda$ , and $\mu$ are specific heat capacity, thermal diffusivity, and laminar viscosity. The first two variables can be computed by Sutherland's law while $c_{p}$ is calculated using quartic polynomial. $D$ with different subscripts are the diffusion coefficients for different processes, and Sc are the corresponding Schmidt numbers. $\operatorname{Pr}_{t}$ is the turbulent Prandtl number. $\overline{\dot{\omega}}_{C}$ is the source term of $\tilde{C}$, which equals the sum of the productivity of $\mathrm{H}_{2} \mathrm{O}$ and $\mathrm{CO}_{2}$.

To calculate the turbulent viscosity $\mu_{t}$ and the Reynold stress $\bar{\tau}_{i j}^{R}$, the shear stress transport (SST) $k-\omega$ model with excellent performance [26] in the supersonic flow is implanted into the code, and the turbulent model has shown good performance in the supersonic mixing flow field in the previous study [24]. For more information about the turbulent model, refer to the study of Li et al. [27].

2.2. Combustion Model and Numerical Schemes. The supersonic turbulent combustion process that occurred in the scramjet is quite difficult to stimulate due to the complex combustion process. A huge computational resource must be paid to simulate the real combustion process. However, the flamelet combustion model $[28,29]$ is able to reduce the computational cost by making a table early no matter how many steps of the reaction mechanism. One of the flamelet combustion models, the FPV model, takes the nonsteady state effect into account when generating the tables and can handle the problem of the local flame quenching and reigniting [30]. Due to the above advantages, the FPV model is widely used, and it is implanted into our code.

In this study, the reaction mechanism of ethylene-air combustion is the San Diego main hydrocarbon mechanism [31] developed by the combustion research group of the University of California, San Diego (UCSD). The mechanism, which contains 39 species and 173 elementary reactions, is a large collection consisting of various detailed chemicalkinetic mechanisms existing in previous studies and can model the combustion of hydrocarbons quite precisely. The mechanism has been widely verified in a lot of studies [32]. Firstly, the laminar flamelet databases are generated by the FlameMaster software [33], and then, the corresponding turbulent flamelet databases can be obtained by statistical aver- aging of the laminar flame surface ensemble. In the current study, ethylene is selected as the fuel, and the progress variable is the sum of the mass fraction of $\mathrm{H}_{2} \mathrm{O}$ and $\mathrm{CO}_{2}$. The species boundary parameters for the oxidizer and fuel side are shown in Table 1. The temperature boundary condition for the fuel side is $264 \mathrm{~K}$, which is the same as the static temperature in Table 1. Due to the compression of the inlet and the effect of the boundary layer, the temperature boundary condition for the oxidizer side is set to $1000 \mathrm{~K}$. The reference pressure is $3 \mathrm{kPa}$ based on the wall pressure measured in the experiment.

The in-house code employs a finite volume method to solve the flow field of the supersonic turbulent combustion. A completely structured grid and the van Leer limiter are used to avoid numerical divergence. The difference scheme in the current research is the widely used advective upstream splitting method (AUSM) scheme. The final results are obtained by a $2^{\text {nd }}$-order time stepping method from the initial flow field. The Courant number is used to control the speed of calculation. It is set to 0.1 in the beginning and then doubles in the process of simulation to avoid divergence. The mass flow rate and the residuals are monitored as indexes of simulation convergence. When the residuals decrease by a thousand times and the total mass flow rate decreases to $0.1 \%$ of the inlet mass flow rate, the flow field is converged. To ensure the stability of simulation, the nonfuel flow field is calculated firstly, and then, the fuel nozzles are turned on to get the nonreacting flow field. The reacting flow field is calculated by patching an area near the fuel injector and cavity, where the pressure is high, the progress variable is 0.5 , and the equivalence ratio is 1 . The quasisteady nonreacting and reacting flow fields are computed through enough iteration steps and then analyzed.

2.3. Computational Grids and Boundary Conditions. The configuration in this article is the axisymmetric model scramjet investigated in a previous study [23]. As shown in Figure 1, the axisymmetric model scramjet consists of an inlet, isolator, fuel injector, cavity, and combustor. The fuel injector contains 16 sonic nozzles uniformly distributed on the wall. The diameter of nozzles is $0.75 \mathrm{~mm}$, and the fuel is injected into the main flow at a $45^{\circ}$ inclination angle to a streamwise direction. The divergent angle of the combustor is $5^{\circ}$. For simplicity, only a quarter of the scramjet is calculated and the symmetry boundary is applied, and the circular nozzles in the experiment are replaced by the rectangular nozzles in order to reduce the size of the mesh as much as possible. Previous research [34] has proved the effect of injector configuration that can be omitted, especially the difference between the circular and rectangular nozzles. It is reasonable and low-cost to replace the circular nozzles with rectangular ones. As Figure 1 displays, three different angles $22.5^{\circ}$ (case-22.5), $45^{\circ}$ (case-45), and $90^{\circ}$ (case-90) are taken into consideration for the investigation of the aft wall angle $\theta$ of the cavity. The whole mesh is a structure mesh whose size is approximately 9.4 million. The mesh is significantly refined near the wall and the jet nozzles. The size of the mesh keeps constant with the variation of the aft wall angle. 
TABLE 1: Inlet and fuel nozzle boundary conditions [23].

\begin{tabular}{lcc}
\hline Parameters & Inlet & Fuel nozzles \\
\hline Mach number, $\mathrm{Ma}$ & 4.5 & 1.0 \\
Total temperature, $\mathrm{T}_{0}(\mathrm{~K})$ & 2196 & 300 \\
Static temperature, $T(\mathrm{~K})$ & 444 & 264 \\
Total pressure, $P_{0}(\mathrm{kPa})$ & 164.7 & 57.1 \\
Static pressure, $P(\mathrm{kPa})$ & 0.55 & 31.4 \\
Mass fraction of $\mathrm{O}_{2}, Y_{\mathrm{O}_{2}}(\%)$ & 23.30 & 6.99 \\
Mass fraction of $\mathrm{N}_{2}, Y_{\mathrm{N}_{2}}(\%)$ & 76.70 & 23.01 \\
Mass fraction of $\mathrm{C}_{2} \mathrm{H}_{4}, Y_{\mathrm{C}_{2} \mathrm{H}_{4}}(\%)$ & 0 & 70.00 \\
\hline
\end{tabular}

The nonslip boundary condition is applied to the wall of the scramjet. The temperature condition of the wall is isothermal and set to $450 \mathrm{~K}$, which is very similar to the static temperature of the inflow. It should be noted that the adiabatic and the room-temperature conditions are both not completely suitable because they are too extreme. And the condition in this article is determined through several numerical tests. The inlet and fuel nozzles are treated as the pressure inlet, and the parameters are shown in Table 1. For the outlet, the parameters can be calculated by the internal cells because of the supersonic feature. The previous study [35] has shown that the RANS method cannot simulate the mixing process between the main flow and the fuel jets very properly in the supersonic flow field. The author mixed some air into the fuel jet to achieve the same mixing level in the experiment [35]. In this study, the same method is applied. For the sonic fuel nozzles, 70 percent of the jets is ethylene while the rest is air. The overall equivalence ratio is 1.06 , the same as the experiment. The premixing of the fuel can promote the mixing process and overcome the shortcoming of the RANS method to a certain extent. In order to keep the injection pressure constant, the area of each nozzle is enlarged to 1.43 times of the original nozzle. The whole flow field is initialized by the inlet flow variables.

2.4. Mesh Independence Verification. To verify the mesh independence, three structural meshes of case-22.5 are employed to simulate the flow field without fuel injection. The sizes of the coarse, medium, and refined meshes are 6.0 million, 9.4 million, and 13.4 million, respectively. As shown in Figure 2, the experimental data is also displayed for comparison and the simulation displays reasonable agreements with the experiments for medium and refined meshes.

Comparing the results of different meshes in Figure 2, it is obvious that the wall pressure of medium and refined meshes shows almost complete agreements with each other while the coarse mesh has some discrepancy compared to the medium and refined ones. The coarse mesh underestimates the pressure hugely inside the cavity. For the position of interaction between the shock and boundary layer near the cavity aft wall, the wall pressure predicted by the coarse mesh is much higher than the other two meshes. It can be inferred that the coarse mesh does not capture the shock preciously. The medium mesh can simulate the flow field with enough accuracy while its computational cost is relatively low. Finally, the medium mesh with a size of 9.4 million is selected in this study, and the mesh keeps the same size among cases with different cavity aft wall angles.

\section{Results and Discussion}

In this part, the major results of the simulation are analyzed and the characteristics of the flow field are discussed. Firstly, the fuel mixing and combustion characteristics of the experimental configuration with an aft wall angle of $22.5^{\circ}$ are investigated and compared with the experimental data. Secondly, the effect of the aft wall angle on the fuel mixing flow field is studied to explore the variation of fuel distribution. Thirdly, the effect of the aft wall angle on the reacting flow field is investigated, and the combustion characteristics are compared among cases with different aft wall angles. Lastly, the effect of the aft wall angle on fuel mixing and combustion characteristics is summarized based on the numerical results.

3.1. Mixing and Combustion Characteristics of CavityAssisted Axisymmetric Scramjet. The experimental scramjet configuration with the cavity aft wall angle of $22.5^{\circ}$ is numerically investigated and analyzed to show the details of the flow field. The results are compared with the experiments to verify the simulation. The nonreacting and reacting flow fields are analyzed, and the main attention is paid to the cavity itself and the shear layer of the cavity.

The numerical and experimental results of wall pressure and exit Mach number are compared in Figure 3. The wall pressure varies with streamwise position $X$ while the Mach number varies with radial position $R$ at the exit of the scramjet. The pressure contour, of which the upper and lower half are nonreacting and reacting flow, respectively, is also displayed to show the flow structure. The simulation results display remarkable agreements with the experiments except for one measurement point inside the cavity in the reacting field. The slight discrepancy between the simulation and the experiment may be caused by the experimental measurement error or the numerical turbulent model. The numerical results of the exit Mach number are consistent with the experimental results except for the area near the wall, where the Mach number is difficult to measure precisely. Therefore, it can be concluded that the numerical simulation predicts the experiment very well.

As the pressure contour in Figure 3 shows, an oblique shock is induced by the inlet, which leads to the increase of pressure. The pressure increases slowly in the isolator because of the development of the boundary layer. The aft wall of the cavity compresses the flow, and a strong shock is induced. The combustion mainly exists downstream of the fuel jets, and the heat release leads to the increase of pressure. The pressure decreases due to the expansion of the divergent combustor, and heat released from the reaction is transformed into gas kinetic energy. The wall pressure of the reacting field is a little higher than that of the nonreacting field, which is in agreement with the experiment. From the above analysis, it can be seen that the simulation can accurately 


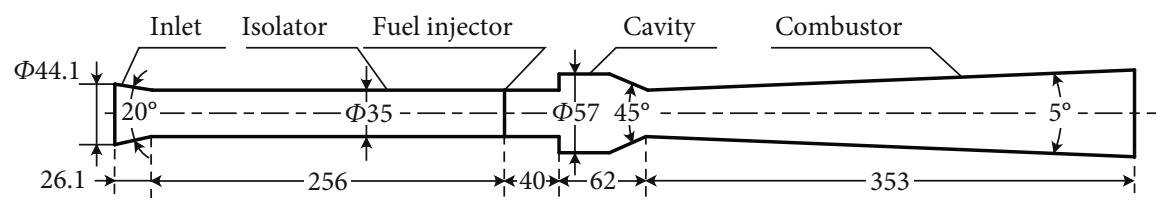

(a)

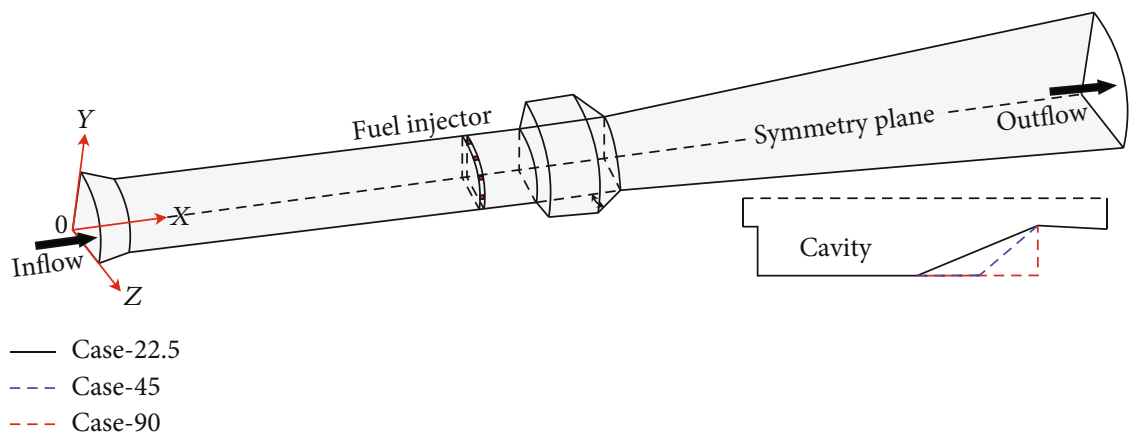

(b)

Figure 1: Schematic of (a) size of the axisymmetric scramjet [23] and (b) one-quarter of the three-dimensional configuration for simulation (units in $\mathrm{mm}$ ).

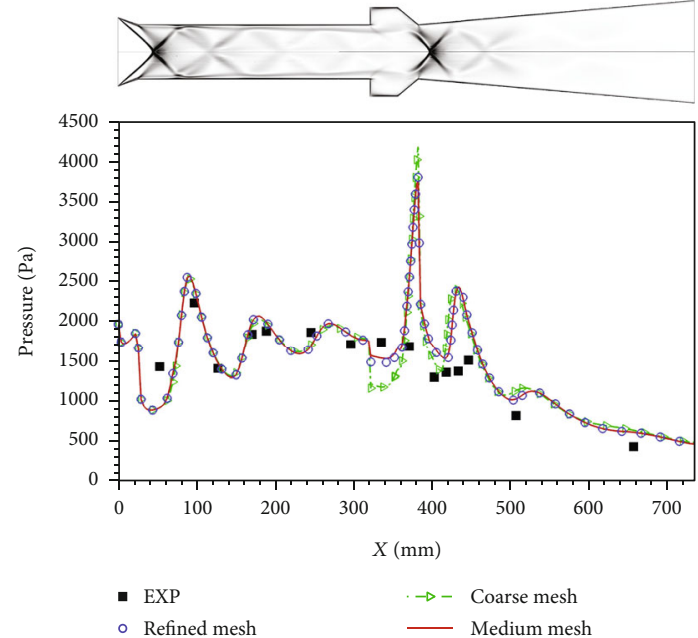

Figure 2: Comparison of wall pressure between experimental [23] and numerical results with different meshes; the gray contour is the numerical Schlieren to show the flow structure and illustrate the variation of the wall pressure.

reflect the characteristics of the axisymmetric scramjet flow field.

Figure 4 displays the distribution of $\mathrm{C}_{2} \mathrm{H}_{4}$ in the nonreacting and reacting flow fields by the streamwise slices. The cavity shear layer is also displayed by the isosurface $\left(\partial U / \partial R=-15000 \mathrm{~s}^{-1}\right)$, and only the isosurface around the cavity is depicted to display the shear layer more obviously. The streamline is colored by the local distance to the axis of symmetry to illustrate the variation of the flow path. In the nonreacting flow field, the fuel is injected from the orifices and gradually diffuses in a circumferential direction. The fuel distribution becomes almost uniform near the front wall of the cavity. The cavity is full of $\mathrm{C}_{2} \mathrm{H}_{4}$ because of the significant mass exchange through the cavity shear layer. In contrast, there is no fuel existing inside the cavity and most of the fuel is consumed upstream of the cavity aft wall in the reacting flow field. Comparing the nonreacting and reacting flow fields, it is obvious that the reaction and heat release change the flow field hugely. In the nonreacting flow field, the cavity shear layer originates from the cavity leading edge and grows gradually, which is the same as the cavity shear layer configuration in the rectangular combustor with a cavity [36]. The streamlines bend to the cavity floor in the middle of the cavity, and the expansion of the cavity results in the transformation of the shear layer. However, the effect of reaction and heat release affect the cavity shear layer in the reacting flow field. The cavity with high pressure is a source of heat, and the main flow does not expand near the cavity. The cavity shear layer, originating from the leading edge, keeps thin and straight. The streamlines are straight and do not bend to the cavity floor, because the heat release is violent and the pressure is high inside the cavity. The decrease of pressure due to the expansion of the flow tunnel is counterbalanced by the increase of pressure due to the reaction and heat release.

The Mach number contour is displayed in several slices in Figure 5. The streamlines and the isoline of sonic speed are displayed to show the flow characteristics. The Mach number increases near the cavity in the nonreaction field because of the expansion of the flow tunnel. The subsonic area inside the cavity is small, and the main flow compresses the recirculation zone. An interesting phenomenon is the normal shock near the axis of symmetry. A strong shock is induced by the compression of the aft wall of the cavity, and the main flow is reduced to subsonic. This is also captured in a previous study [14] and may cause a lot of loss of total pressure. The shock near the axis of symmetry disappears in the reacting field. The area of the subsonic area inside the cavity increases, and the velocity of the main flow keeps almost constant near the cavity. There exists a subsonic 


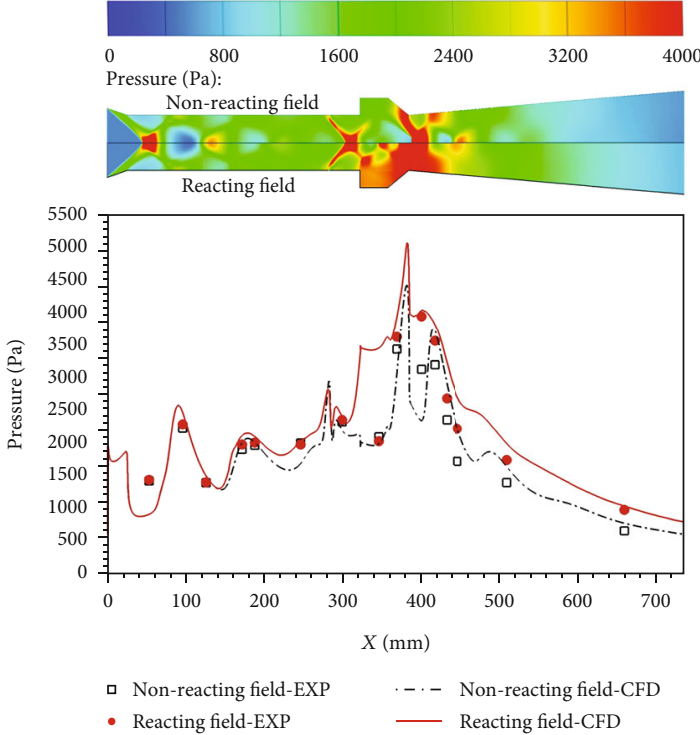

(a)

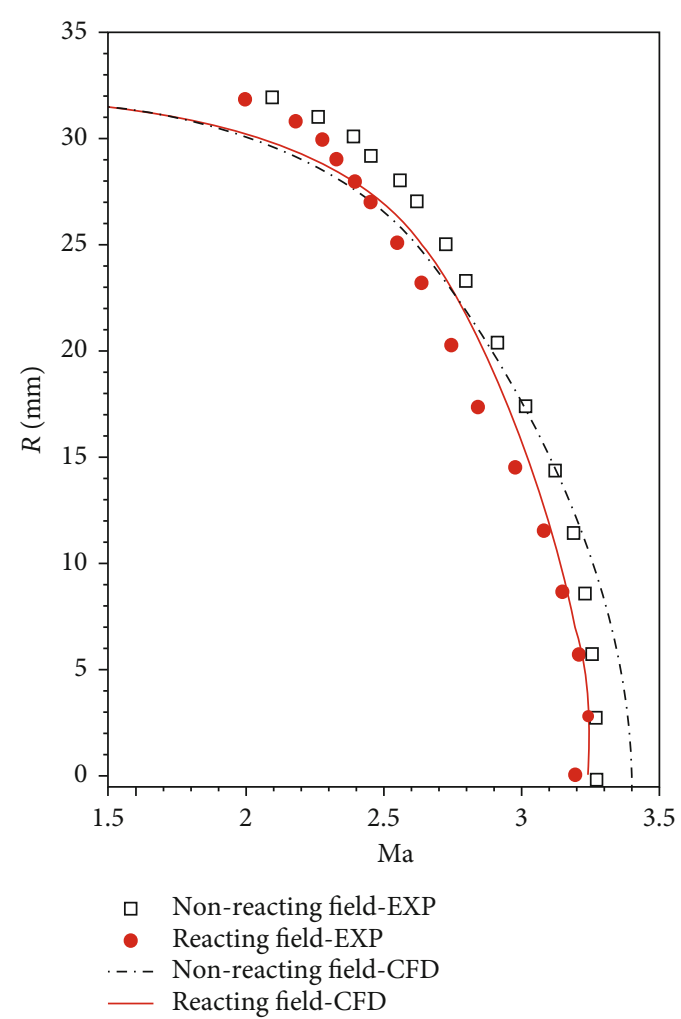

(b)

FIgURe 3: (a) Wall pressure and (b) exit Mach number comparison between numerical and experimental results [23] for the nonreacting and reacting flow fields; the pressure contour is also displayed to show flow structures.

zone upstream of the cavity shown by the pink line due to the separation of the boundary layer that induced the increase of pressure in the cavity.

In the scramjet, the cavity can provide hot products and radicals to the remaining mixture of fuel and air. The mass exchange rate of the fuel occurs through the cavity shear layer and is important to hold the flame. The fuel mass exchange rate across the cavity shear layer has been studied by previous research [37]. As shown in Figure 6, the exchange velocity $V_{r}$ is the velocity perpendicular to the lip surface of the cavity.

The fuel mass exchange rate for both nonreacting and reacting flow fields at the cavity lip surface is displayed in Figure 6. The fuel mass exchange rate near the cavity shear layer displays axisymmetric characteristics in the nonreacting flow field. The value of fuel mass exchange rate in the same streamwise direction almost keeps constant in the different circumferential places. Due to the flow expansion in the front part of the cavity, a great deal of fuel enters into the cavity through the cavity shear layer. At the end of the cavity, the fuel exits from the cavity due to the effect of the cavity aft wall. The mass exchange is violent, and the magnitude is on the order of one-tenth. In contrast, the mass exchange is moderate, and the magnitude is on the order of one-thousandth in the reacting flow field. There are two reasons explaining this phenomenon. One is that a large part of the fuel is consumed in the reacting flow field, and the other is that the combustion and heat release affect the flow field and change the characteristics of mass exchange through the cavity shear layer. As shown in Figure 4, the configuration of the cavity shear layer is changed by the reaction and heat release in the reacting flow field. However, the fuel also enters into the cavity in the front part of the cavity lip surface and exits in the back part. Another interesting phenomenon is that the fuel mass exchange rate near the cavity shear layer displays nonaxisymmetric characteristics in the reacting flow field. The areas where the fuel enters into the cavity concentrate to two main places in the circumferential direction, and this may result in nonaxisymmetric combustion.

From the above analysis, it can be concluded that combustion makes a great effect on the flow field. The cavity shear layer of the axisymmetric cavity shows some similarities to the rectangular cavity in the nonreacting flow field. However, because of the reaction and heat release, the flow structures change hugely in the axisymmetric reacting cavity flow. This may result from the relatively bigger volume of the cavity in the axisymmetric scramjet when the area of the inlet is the same as the rectangular scramjet.

3.2. Effect of Aft Wall Angle on the Nonreacting Flow Field. A series of optimizations should be carried out to find the most advantageous configuration before a new scramjet is surely applied to engineering. The effect of the aft wall angle of the cavity on the nonreacting flow field should be investigated to find an optimized cavity flameholder. As mentioned 


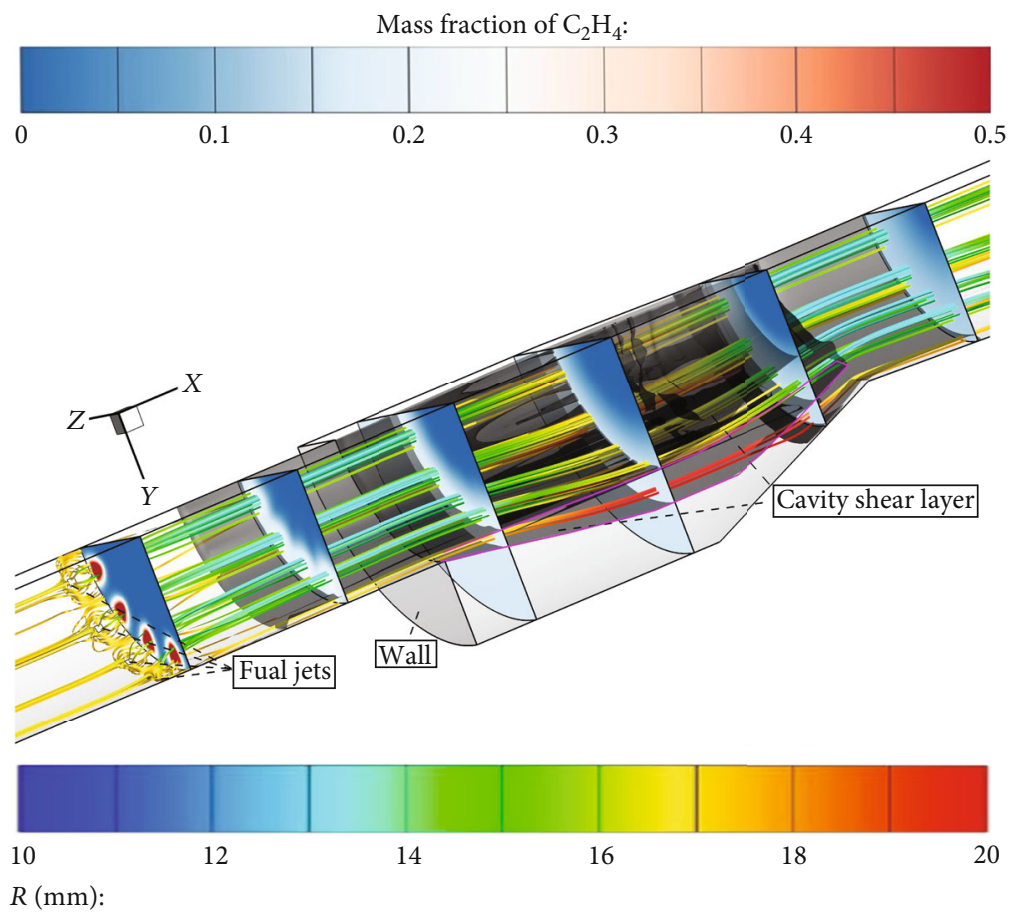

(a)
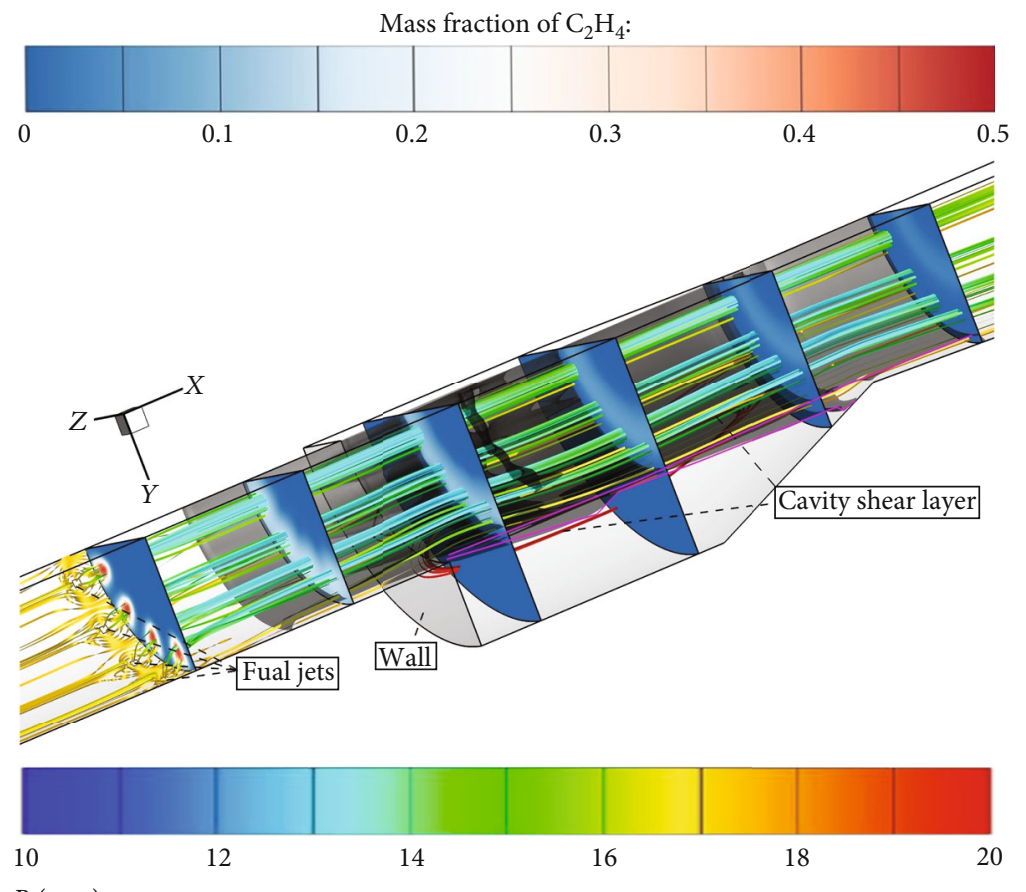

$R(\mathrm{~mm})$ :

(b)

FIGURE 4: Fuel distribution of the (a) nonreacting and (b) reacting flow fields of case-22.5; the streamline is colored by the local distance to the axis of symmetry, and the isosurface is defined as the surface where the partial derivative in the radial direction of streamwise velocity $\partial U / \partial R$ equals $-15000 \mathrm{~s}^{-1}$ to display the boundary of the cavity shear layer.

in Subsection 2.3, the label "case-angle" is used to indicate the cavity aft wall angle in this article. For example, case-22.5 refers to the configuration with the cavity aft wall angle of $22.5^{\circ}$. The way of changing the cavity aft wall angle is adopted in previous research [36]. The only variable, the cavity aft wall angle $\theta$, is defined as the angle between the cavity aft wall and the streamwise direction. Based on the original configuration with the aft wall angle of $22.5^{\circ}$, two more configurations with different angles $\left(45^{\circ}\right.$ and $\left.90^{\circ}\right)$ are investigated. The cavity length $L=62 \mathrm{~mm}$, defined as the maximal length 

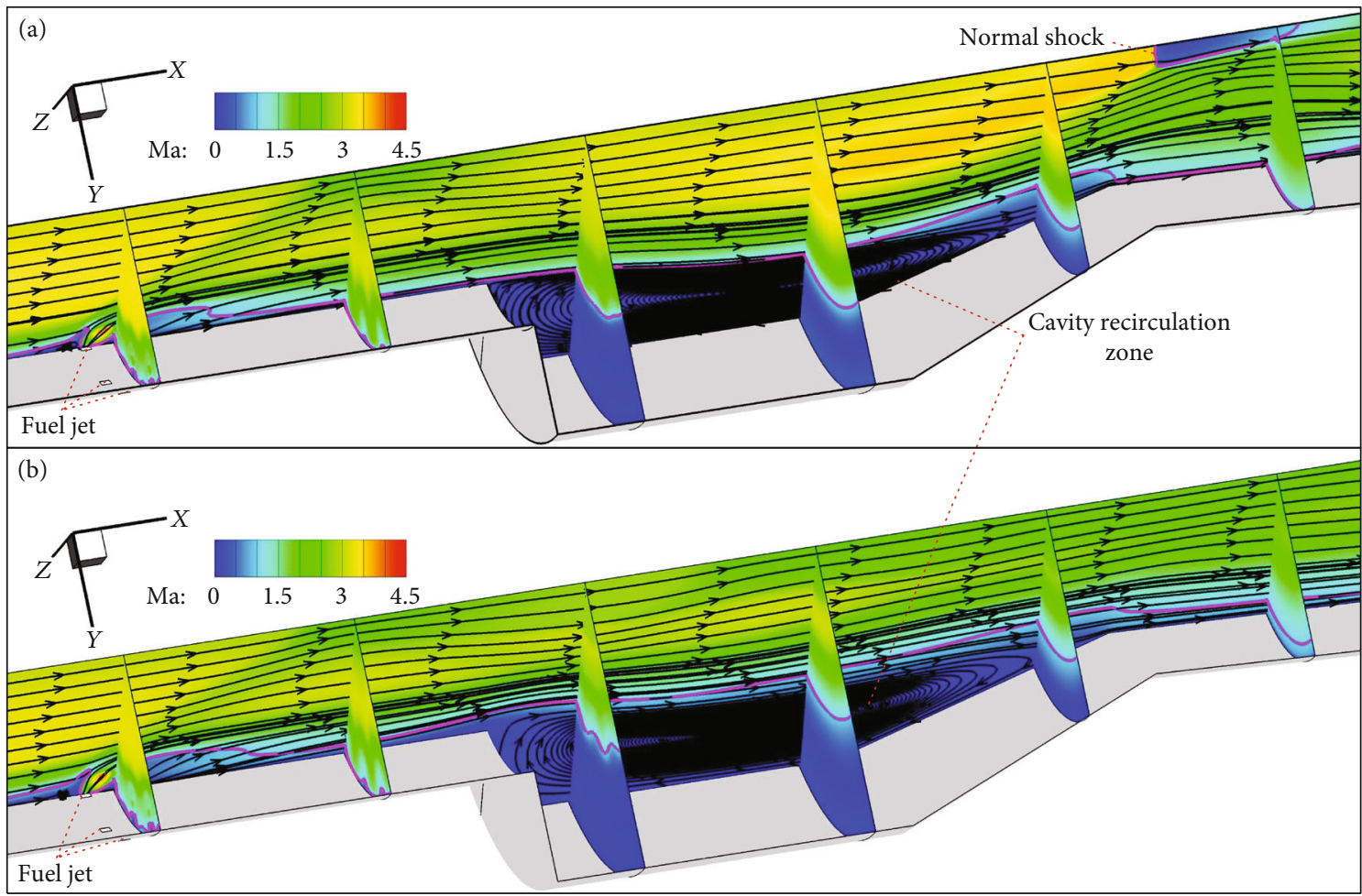

FIGURE 5: Slices of Mach number contour of (a) the nonreacting and (b) reacting flow fields of case-22.5; the pink line is the line of sonic speed.

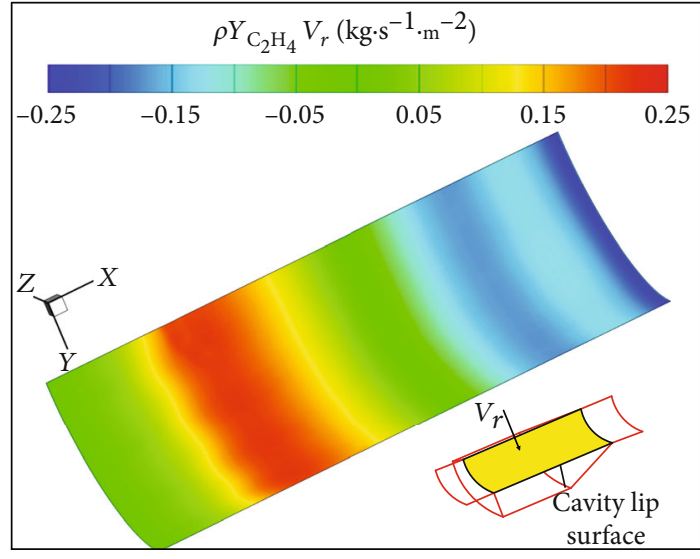

(a)

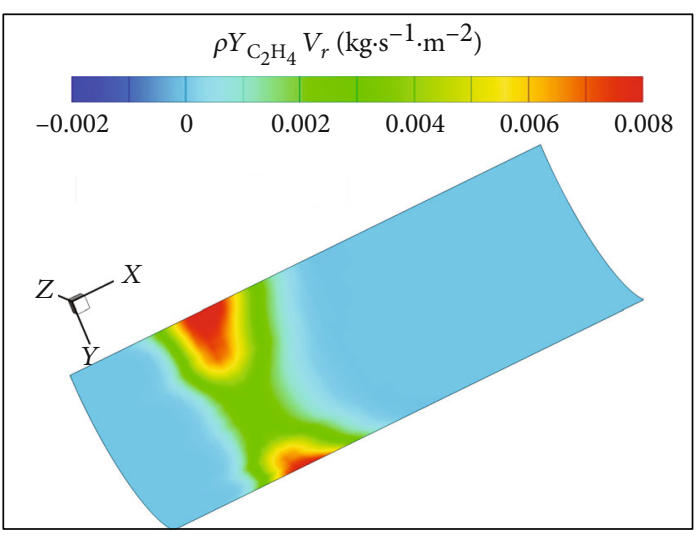

(b)

FIGURE 6: The cavity fuel mass exchange rate of the (a) nonreacting and (b) reacting flow fields of case-22.5; the cavity lip surface is displayed as the yellow surface in the schematic diagram in (a).

in the streamwise direction, keeps constant with the variation of the cavity aft wall angle.

In order to explore flow characteristics, the flow structures of configurations with different aft wall angles are displayed in Figure 7 by the Mach number contour. In each figure, the upper half (labeled by "jet plane") is the slice which goes through the center of the injection orifice while the lower half (labeled by "nonjet plane") is the slice which goes across the center between two injection orifices. The same labels used in the rest of the article represent the same planes in Figure 7. As shown in the figure, the nonreacting flow field of case-22.5 is completely different from others. When the aft wall angle of the cavity is small, the flow expands and speeds up near the cavity seriously. The Mach number increases near the center of the cavity, and then, a normal shock is induced by the compression of the cavity aft wall. The same shock structure was also observed in the simulation of axisymmetric cavity flow with the same aft wall angle in a previous study [14]. Due to strong normal shock, the main flow slows down downstream of the cavity. For case-45 and case-90, the Mach number of the main flow increases a little near the cavity and decreases slightly after passing the cavity. At the combustor, the flow speeds up for all cases due to the expansion of the wall. The speed of the main flow of case- 45 


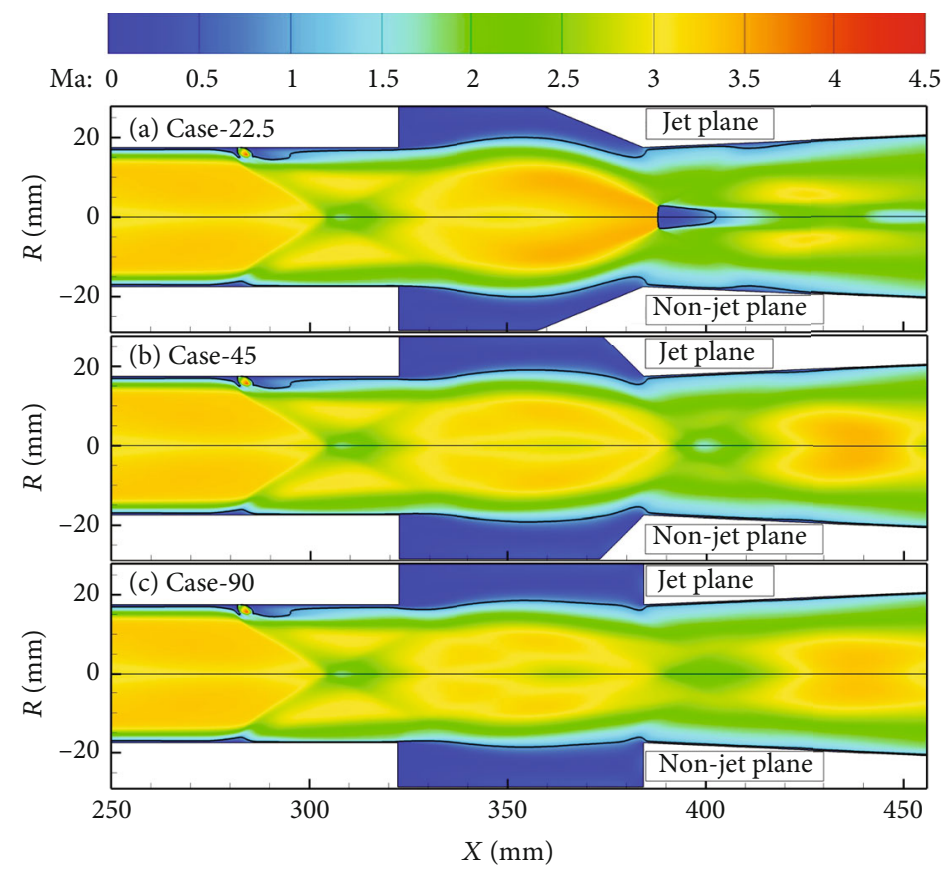

Figure 7: Mach number contours of all cases in the nonreacting flow field; the black solid line is the isoline of sonic speed.

and case- 90 is greater than that of case- 22.5 between $400 \mathrm{~mm}$ and $450 \mathrm{~mm}$.

The partial derivative in the radial direction of the streamwise velocity $\partial U / \partial R$ is displayed in Figure 8 to show the variation of the cavity shear layer under different aft wall angles. The cavity shear layer plays an important role in promoting the mixing of the fuel and holding the flame in supersonic flow. For all cases, the configuration of the cavity shear layer in the jet plane and nonjet plane is almost the same to each other, which indicates that the fuel jets make little effect on the cavity shear layer. However, the cavity shear layer changes a lot with the variation of the aft wall angles. For case-22.5, the cavity shear layer starts from the cavity front wall and becomes thick in the middle of the cavity. The shear layer bends to the cavity bottom because of the expansion of the flow and separates from the aft wall because of the compression. When the aft wall angle increases, the cavity shear layer keeps almost straight and attaches to the cavity aft wall. For case-45 and case-90, the cavity shear layer becomes thin downstream of the middle of the cavity. An interesting phenomenon is that there exists an isolated part of the shear layer in case- 45 as the boundary of the cavity shear layer shows in Figure 8. It seems that a part of the cavity shear layer falls off the main part when the aft wall angle increases. The above phenomenon was also discovered in the rectangular scramjet, and previous research [36] found that the cavity shear layer bends more seriously to the cavity bottom with the decreasing of the cavity aft wall angle.

For the cavity-assisted scramjet, the variation of the cavity shear layer will affect the fuel distribution and then make a difference to the combustion and the overall performance. It is worthwhile to investigate the fuel mixing characteristics for all cases. In Figure 9, four $X$-direction slices are contoured by a mass fraction of $\mathrm{C}_{2} \mathrm{H}_{4}$ to show the distribution of the fuel.
The isoline of the appropriate equivalence ratio is marked by the black line while the blue circle is the lip line of the cavity. For all cases, the cavity is full of fuel and the environment is fuel-rich. The surplus fuel may lead to weak combustion inside the cavity and weaken the effect of the cavity on flameholding. The fuel concentrates near the fuel jets on the slice $X=325 \mathrm{~mm}$ and gradually diffuses to both sides of the cavity lip in the downstream slices. The penetration depth of the fuel is large in the first slice and then decreases in the second and third slices. In the last slice, the penetration depth to the main flow increases again. This phenomenon is very similar to the results of jet in a supersonic cross-flow in a previous study [38]. Comparing the fuel distribution of cases with different aft wall angles, it can be concluded that the smaller the aft wall angle is, the higher the fuel concentration is inside the cavity. This may be because the volume of the cavity increases and the concentration of $\mathrm{C}_{2} \mathrm{H}_{4}$ decreases, as the aft wall angle increases. From this view, a large aft wall angle may promote the mixing of fuel and make a benefit to the combustion.

In order to investigate the fuel distribution quantitatively, the mass fraction of $\mathrm{C}_{2} \mathrm{H}_{4}$ at different lines in the jet plane is shown in Figure 10. The dashed black line is the cavity lip, and the red solid line is used to indicate the fuel boundary where the mass fraction of $\mathrm{C}_{2} \mathrm{H}_{4}$ equals 0.01 . Corresponding to Figure 9, the fuel concentration inside the cavity decreases as the aft wall angle increases. However, the fuel concentration outside the cavity increases as the aft wall angle increases. It can be concluded that as the aft wall angle increases, the fuel distributes more uniformly around the cavity. As the red solid line shows, the fuel penetrates more deeply into the main flow when the aft wall angle increases. The increase of fuel penetration depth is implicit in line $X$ $=340 \mathrm{~mm}$ and becomes very obvious in line $X=360 \mathrm{~mm}$ 


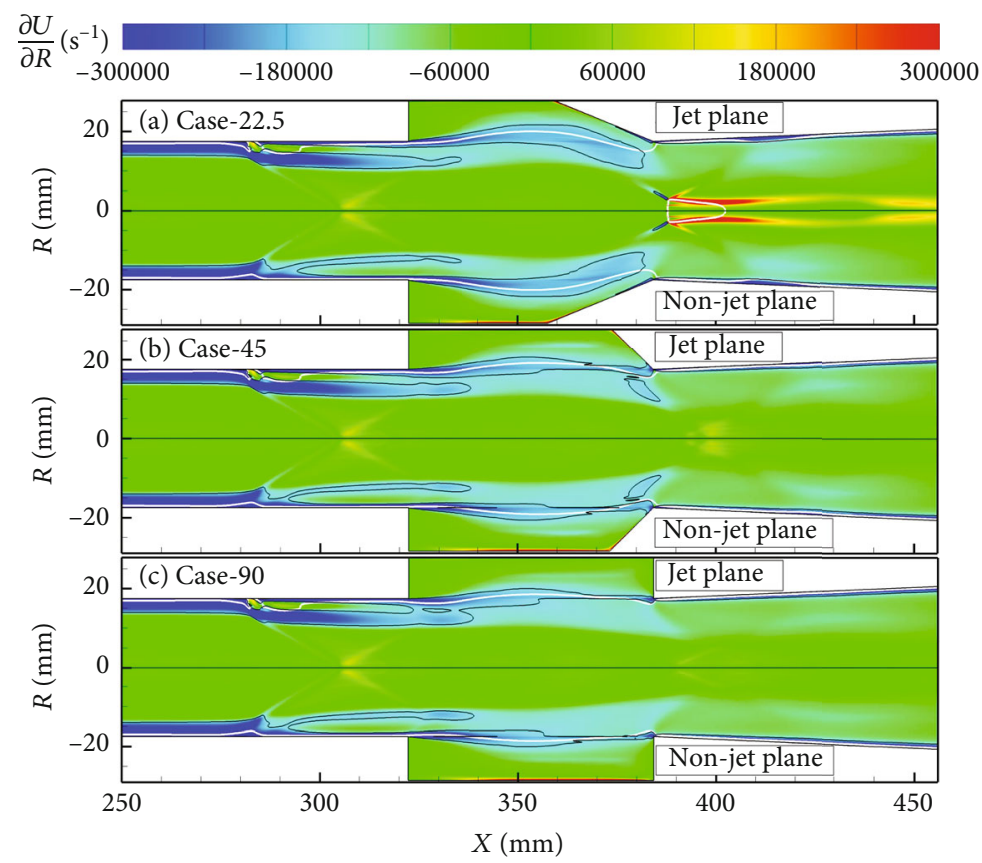

FIGURE 8: Contours of streamwise velocity partial derivative in the radial direction of all cases in the nonreacting flow field; the white solid line is the isoline of sonic speed; the boundary of the cavity shear layer is marked by a black solid line where $\partial U / \partial R=-15000 \mathrm{~s}^{-1}$.

and $X=380 \mathrm{~mm}$. The above analysis shows that a large cavity aft wall angle improves the mixing process of the fuel, and this could result from the large volume of the cavity.

The combustion is controlled by the fuel mixing in the scramjet, and the fuel mixing efficiency $\left(\eta_{\text {mix }}\right)$ indicates the mixing performance of the scramjet. $\eta_{\text {mix }}$ is the mass flow rate ratio of the burnable fuel to the total fuel at a certain streamwise slice. The strict definition of $\eta_{\text {mix }}$ exists in previous research [39]. Figure 11 shows the fuel mixing efficiency for all configurations. The cavity is the main part to enhance the mixing process of the fuel and hold the flame, so most of the attention in this article is paid to the cavity and only the mixing efficiency near the cavity is displayed in Figure 11. It is very clear that with the increasing of the cavity aft wall angle, the mixing efficiency improves gradually. This conclusion is consistent with what is found in the simulation of the rectangular combustor with a cavity in a previous study [36]. A larger cavity aft angle is able to induce the instabilities of the cavity shear layer, which further results in a rapid mixing process. This indicates that there are some similarities between the rectangular and axisymmetric scramjets.

3.3. Effect of Aft Wall Angle on the Reacting Flow Field. Some important phenomenon has been revealed by the detailed investigation of the effect of the aft wall angle on the nonreacting flow field. In order to further investigate the effect of the aft wall angle on the combustion process, the reacting flow fields of different cases are analyzed to show the effect of the aft wall angle on the combustion and flameholding characteristics.

Figure 12 displays the Mach number contour of the reacting flow field for all cases. Affected by the combustion and heat release of reaction, the reacting flow field is completely different from the nonreacting flow field. Because of the heat of the reaction, the speed of the main flow decreases downstream of the fuel jets. For all cases, the flow does not expand to the cavity because the cavity itself is high-pressure and full of hot production of the reaction. The area of the subsonic zone around the cavity increases compared to the nonreacting flow field. The speed of the main flow increases again downstream of the cavity. The effect of the aft wall angle is obscure in the reacting flow field, and all the Mach number contours of different cases are the same as each other.

In the supersonic reacting flow field, the cavity shear layer is of vital importance for flame stabilization and effective combustion. Therefore, Figure 13 displays the fuel mass exchange rate near the cavity shear layer of the reacting flow field for all cases to show the interaction between the cavity and the main flow. The black solid line is a boundary to mark the area where the fuel mass exchange rate is higher than $0.0005 \mathrm{~kg} \cdot \mathrm{s}^{-1} \cdot \mathrm{m}^{-2}$. The distribution of the fuel mass exchange rate shows some similarities among cases with different aft wall angles, but the magnitude varies hugely with the increase of the aft wall angle. It is obvious that the fuel mass exchange rate decreases a lot when the aft wall angle increases. It is indicated that with the increase of the cavity aft wall angle, the cavity itself tends to become independent of the main flow and the interaction between the cavity and the main flow becomes weak. However, the cavity with a large aft wall angle has a big volume, which can provide more free radical and heat for the rest of the fuel-air mixture. Therefore, the reacting flow field should be investigated further to explore the effect of the aft wall angle.

In order to show the effect of reaction heat release, Figure 14 displays the temperature contours in the jet plane and the nonjet plane for cases with different aft wall angles. 


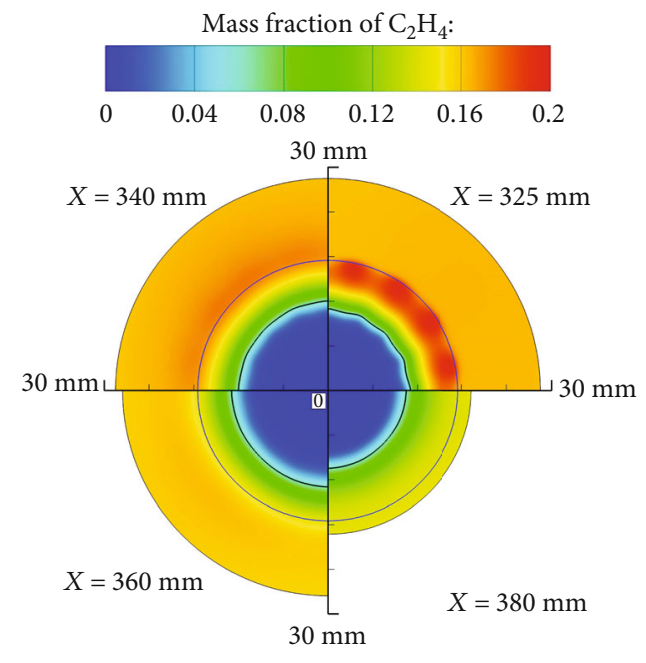

(a)

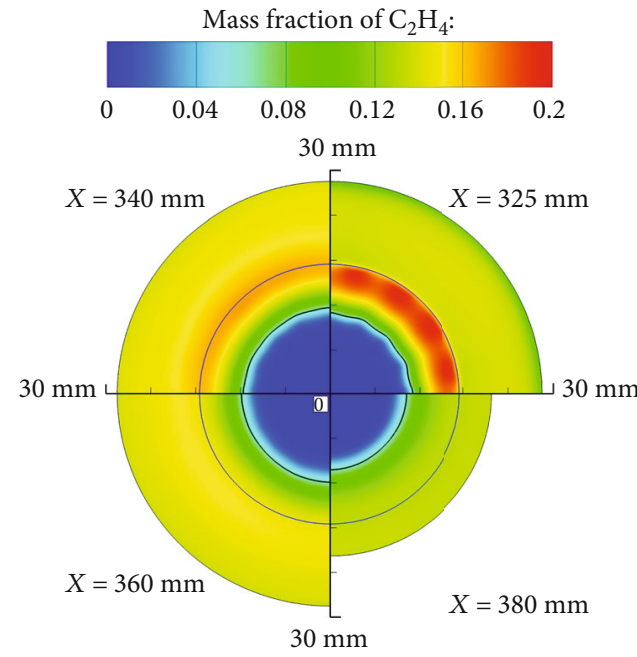

(b)

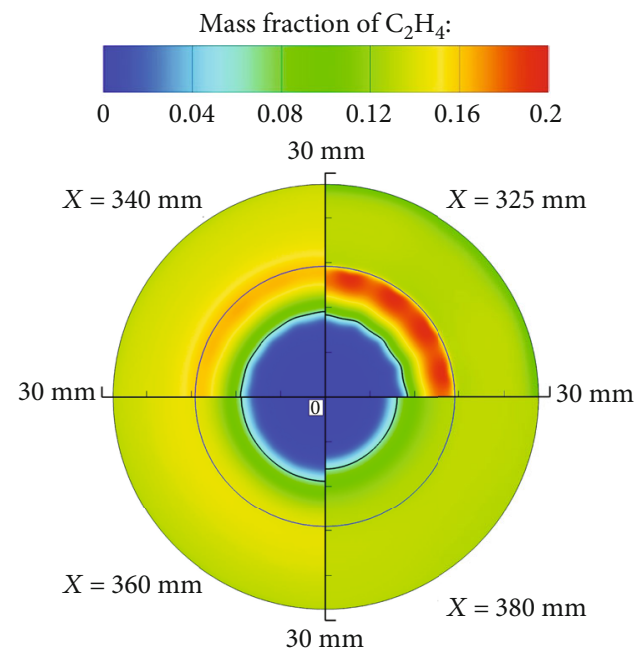

(c)

FIGURE 9: $X$-direction slices of mass fraction of $\mathrm{C}_{2} \mathrm{H}_{4}$ contour for all cases in the nonreacting flow field; the black and blue solid lines are the isoline of the appropriate equivalence ratio and the lip line of the cavity, respectively.

For case-22.5, the high-temperature zone mainly concentrates in the aft wall of the cavity. The temperature in the near-wall region downstream of the cavity is also very high, because the product of the reaction flows out of the cavity. In the region near the front wall of the cavity, the temperature is relatively low. This may be because excessive fuel enters into the cavity owing to the strong interaction between the cavity and main flow. As the aft wall angle increases, the area of the high-temperature region inside the cavity becomes bigger. For case-90, the temperature of almost the whole cavity is pretty high, and this is beneficial to flame stabilization and robust combustion. Although Figure 13 shows that the interaction between the cavity and main flow tends to be reduced with the increase of the aft wall angle, the temperature of the cavity is higher for the case with a bigger aft wall angle. Therefore, the effect of the cavity aft wall angle on the combustion in the axisymmetric scramjet is not yet very clear. Another interesting phenomenon is that the com- bustion mode of the current axisymmetric scramjet is very similar to the combined cavity shear-layer/recirculation stabilized combustion mentioned in previous research [40]. The combined cavity shear-layer/recirculation stabilized combustion is the most robust combustion mode in the rectangular scramjet [40], and this indicates that the axisymmetric scramjet has some advantages over the rectangular scramjet.

The combustion efficiency $\left(\eta_{\text {com }}\right)$ is a crucial quantitative index to show the capability of the scramjet. $\eta_{\text {com }}$ is the mass flow rate ratio of the consumed fuel at different streamwise planes to the total fuel from the fuel nozzles [18]. High combustion efficiency means that the chemical energy of the fuel is almost completely turned into internal energy of the gas. The combustion efficiency of all cases is shown in Figure 15. The main attention is also paid to the area near the cavity. It is very obvious that the combustion efficiency improves when the aft wall angle increases. It indicates that 


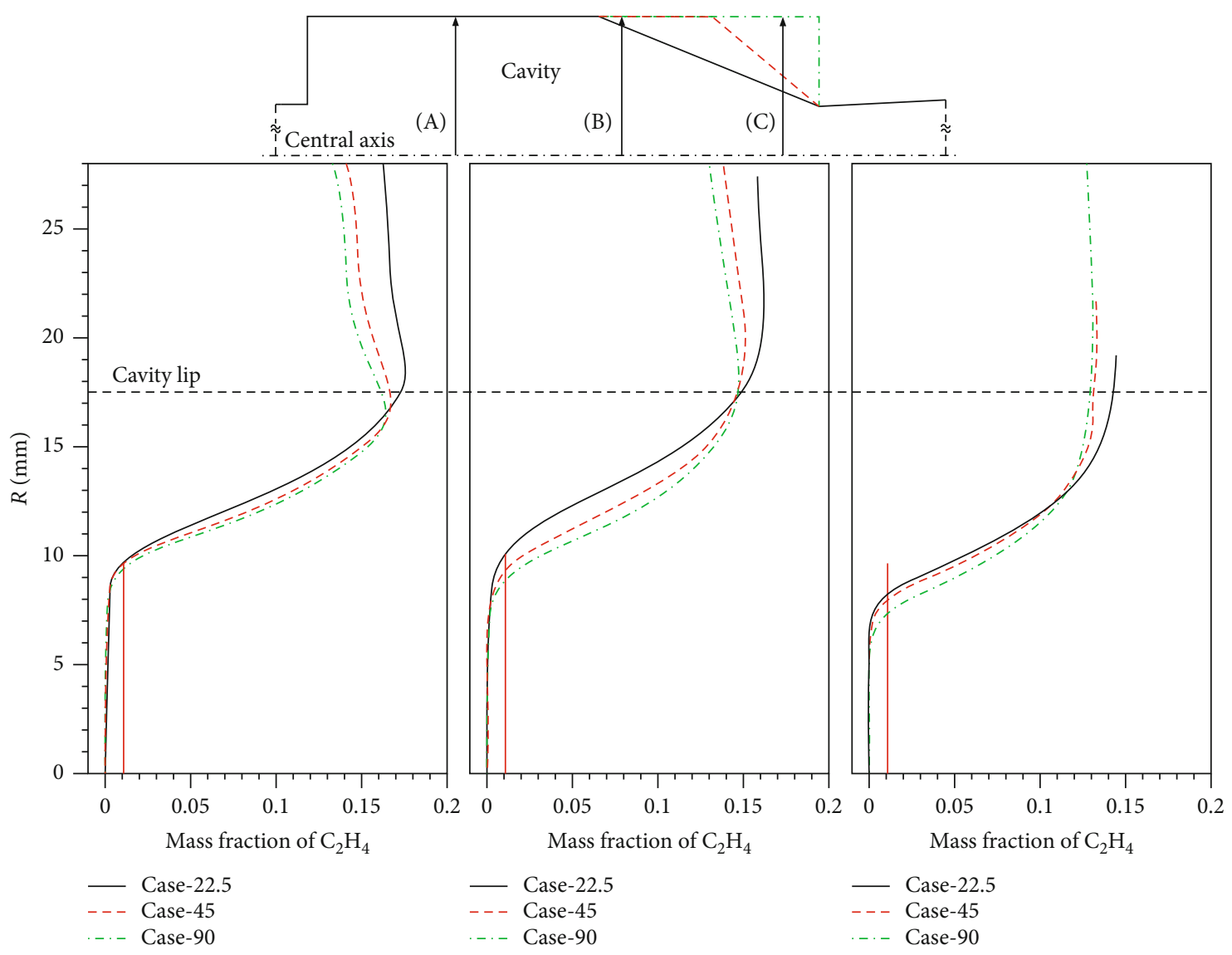

(a) $X=340 \mathrm{~mm}$

(b) $X=360 \mathrm{~mm}$

(c) $X=380 \mathrm{~mm}$

Figure 10: Mass fraction of $\mathrm{C}_{2} \mathrm{H}_{4}$ at different lines in the jet plane; the black dashed line is cavity lip, and the red solid line is fuel boundary where the mass fraction of $\mathrm{C}_{2} \mathrm{H}_{4}$ equals 0.01 .

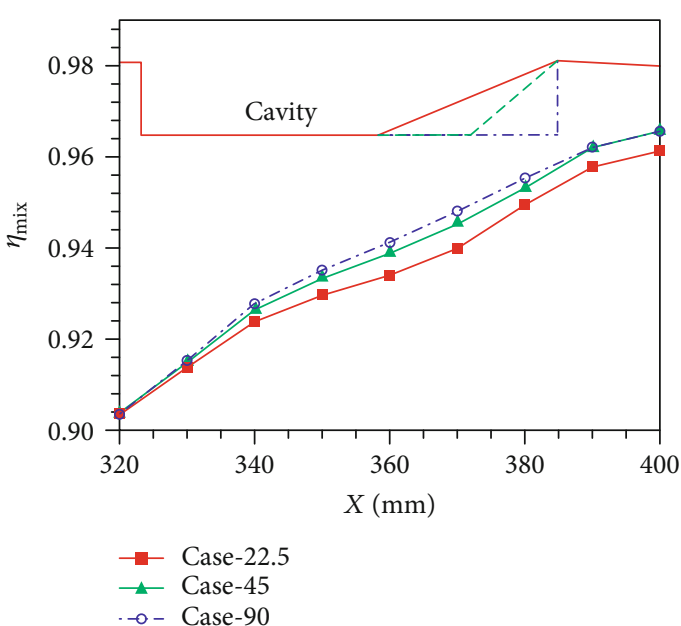

FIGURE 11: Variation of fuel mixing efficiency around the cavity for all cases.

more chemical energy of the fuel is released, which results in higher combustion efficiency, when the aft wall angle is larger. The quantitative comparison shows that stronger combustion happens in cases with larger aft wall angles. The above analysis has shown that the fuel mass exchange rate through the cavity lip surface decreases with the increase of the aft wall angle. This may lead to a weak interaction between the main flow and cavity and further do harm to the combustion. However, the size of the high-temperature area inside the cavity increases with the increase of the aft wall angle as shown in Figure 14. The high-temperature cavity can provide more energy for the unburned mixture of fuel and air, and this can enhance combustion performance. The above two factors compete with each other when the aft wall angle increases. The improved combustion efficiency indicates that the effect of the larger high-temperature area inside the cavity (Figure 14) weighs more than the effect of the weaker interaction between the cavity and the main flow (Figure 13), when the cavity aft wall angle increases. From this view, we can conclude that the combustion performance improves when the aft wall angle increases. This conclusion is the same as previous research of the rectangular cavityassisted scramjet with different aft wall angles [36]. This further indicates that there exist some similarities between scramjets with different inlet configurations.

3.4. Mixing and Combustion Characteristic Analysis. The nonreacting and reacting flow fields of cases with different aft wall angles have been discussed, and some interesting and important characteristics of these fields have been 


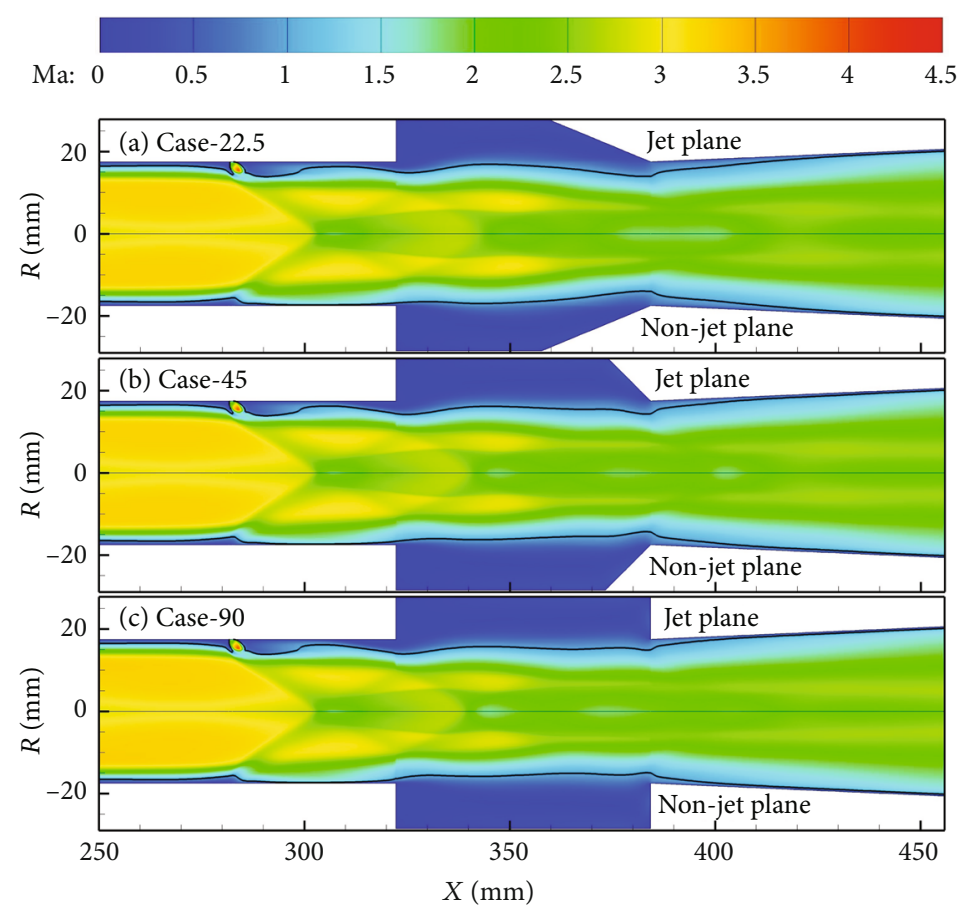

FIGURE 12: Mach number contours of all cases in the reacting flow field; the black solid line is the isoline of sonic speed.

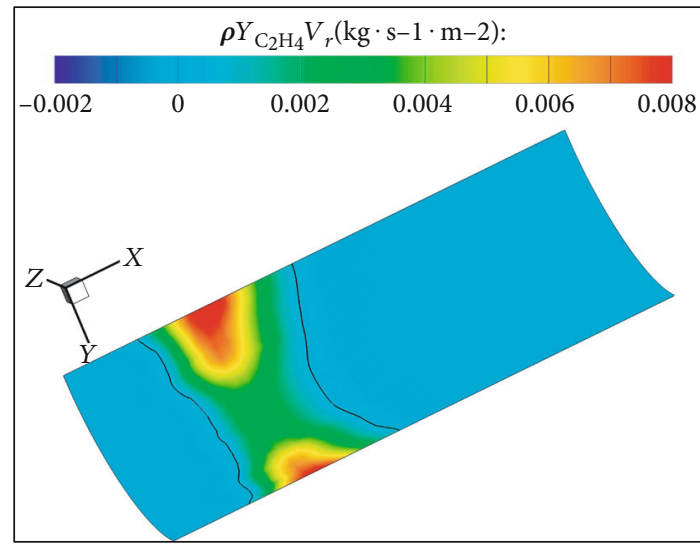

(a) Case-22.5

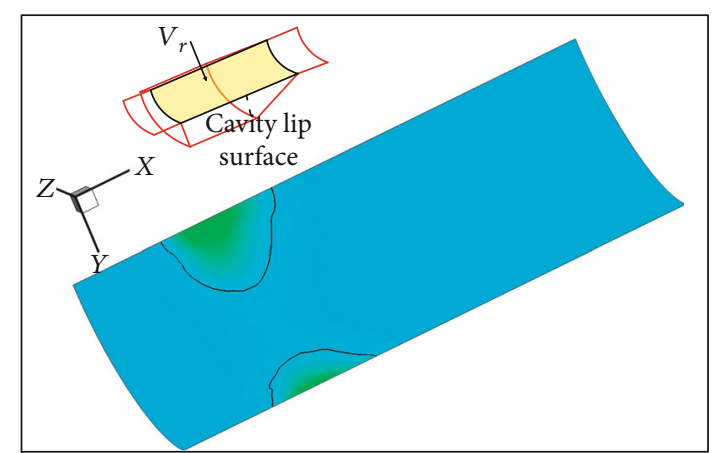

(b) Case-45

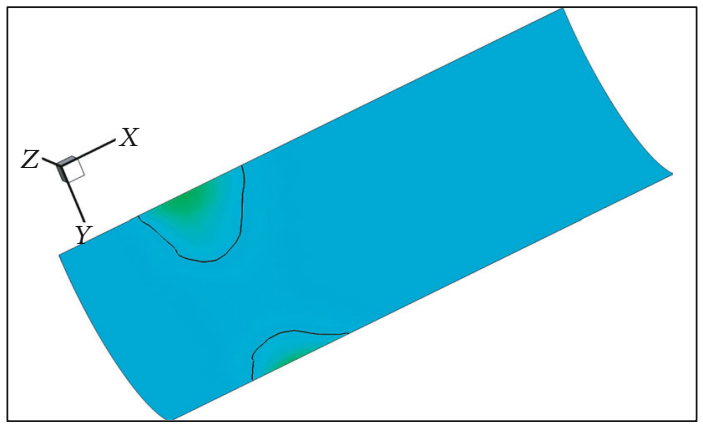

(c) Case-90

FIGURE 13: The cavity fuel mass exchange rate for all cases; the cavity lip surface is displayed as the yellow surface in the schematic diagram in (b).

uncovered above. In this subsection, the main fuel mixing and combustion characteristics of the current axisymmetric model scramjet are analyzed to summarize the effect of the aft wall angle on the performance of the scramjet. As Figure 16 shows, the upper half is the schematic of the nonreacting flow field while the lower half is the schematic of 


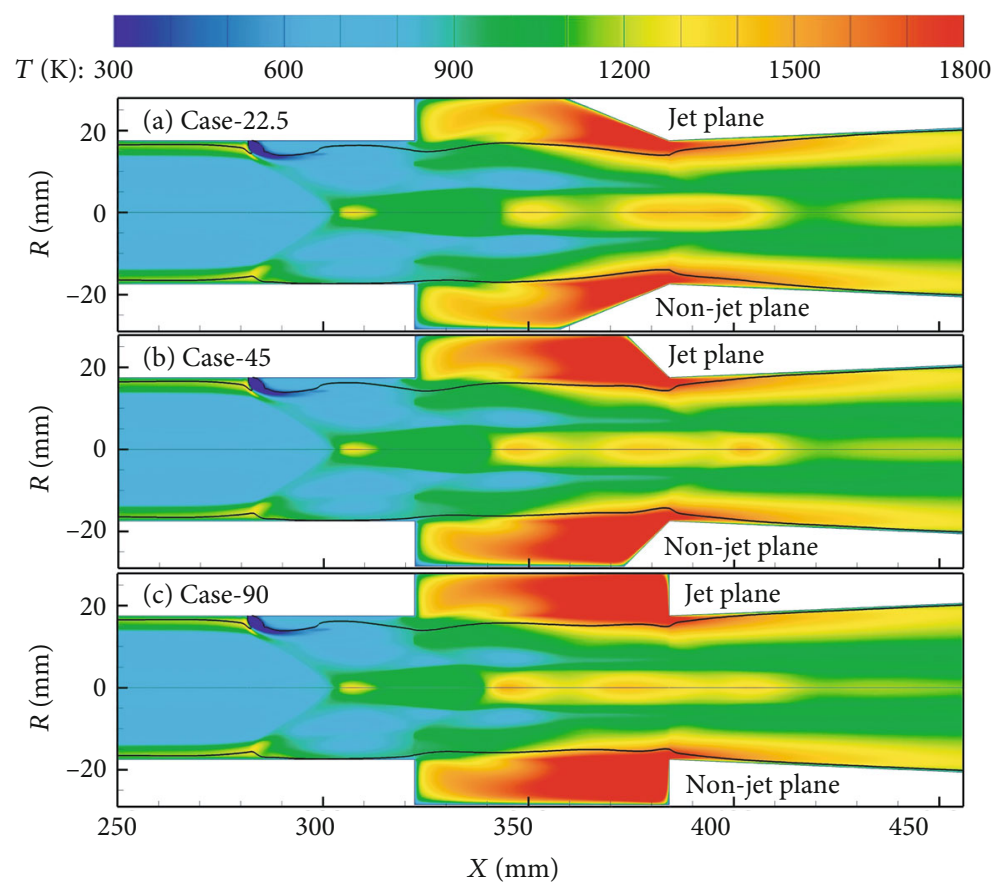

FIgURE 14: Temperature contours of all cases in the reacting flow field; the black solid line is the isoline of sonic speed.

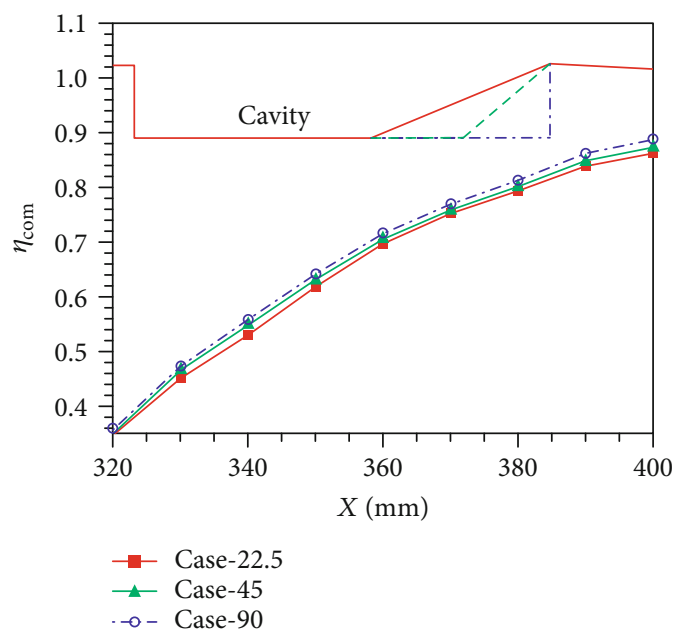

FIGURE 15: Variation of fuel combustion efficiency around the cavity for all cases.

the reacting flow field. It should be noted that the cavity is the most important part of the cavity-assisted scramjet. Therefore, the schematic mainly displays the characteristics of the cavity.

For the fuel mixing process of the nonreacting flow field, the cavity shear layer changes obviously with the variation of the aft wall angle. When the aft wall angle increases, the thickness of the cavity shear layer decreases. For case-22.5, the center of the cavity shear layer bends to the cavity floor and the main flow expands to the cavity. Therefore, most of the fuel is entrained into the cavity and the mixing between the fuel and the air of the main flow is reduced. As Subsection 3.2 shows, most of the fuel concentrates inside the cavity and the mixing efficiency of the fuel are low. The performance of the whole scramjet may become poor due to the bad mixing process for case-22.5. For case-45 and case-90, the expansion of the main flow decreases gradually. More fuel is entrained by the main flow and mixed with the air. As a result of the flow mechanism, the fuel distributes more uniformly around the cavity and the mixing efficiency improves. In conclusion, the fuel mixing performance of the scramjet improves as the aft wall angle of the cavity increases. The phenomenon uncovered in this article is very similar to the conclusion in the previous study [36], but the latter investigates the flow field of the rectangular scramjet.

For the combustion process of the reacting flow field, the interaction between the cavity and the main flow decreases with the increase of the aft wall angle. The cavity is the pool of heat and free radical. Therefore, the reduced interaction between the cavity and the main flow may result in poor combustion performance. On the other hand, the volume of the cavity increases when the aft wall angle increases. Bigger cavity will provide more heat and free radical, and strong combustion will be achieved. What is more, the numerical results show that the temperature of the cavity will increase and the area of high-temperature region becomes bigger. Therefore, there exists competitive relation between the reduced interaction and the larger volume of the cavity. The quantitative indicator, combustion efficiency, shows that the combustion performance will improve when the aft wall angle increases. This implies that the effect of larger volume of the cavity weighs more than the effect of the reduced interaction between cavity and main flow. The numerical results of the reacting flow field in the current study are pretty the same as the previous study [36] on the rectangular scramjet.

The above analysis shows that a larger aft wall angle could improve the performance of the fuel mixing and combustion process for the axisymmetric scramjet. Therefore, it 

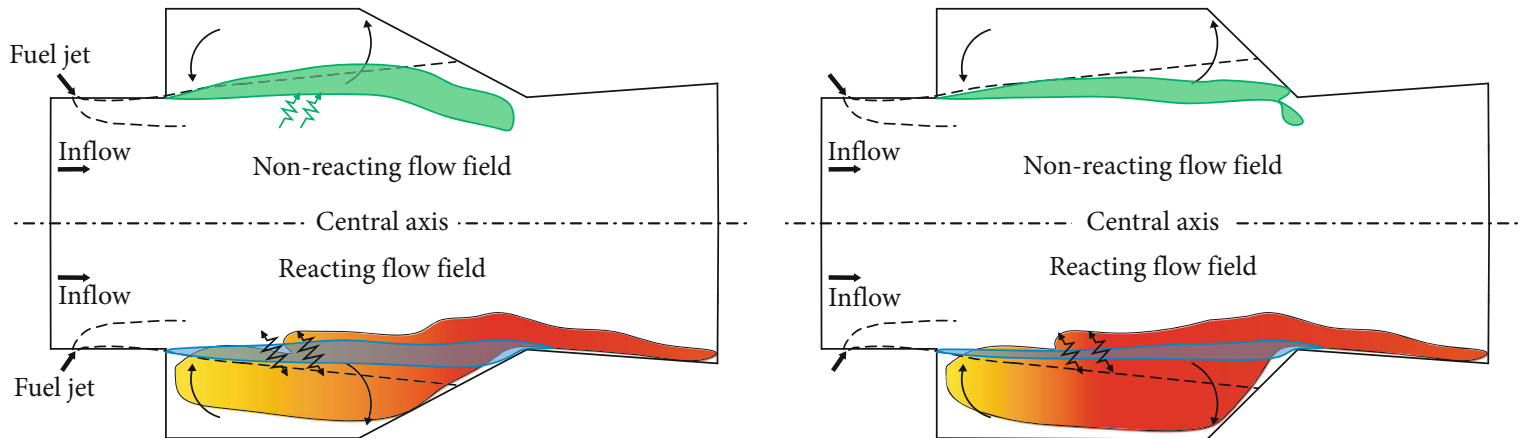

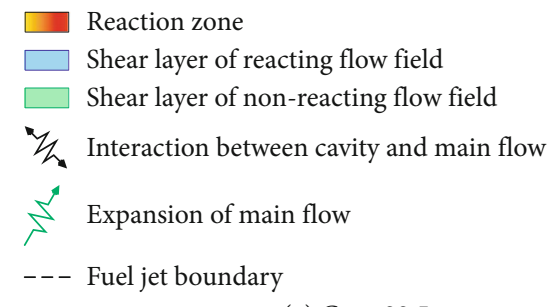

(a) Case-22.5
Reaction zone

Shear layer of reacting flow field

Shear layer of non-reacting flow field

Interaction between cavity and main flow

5 Expansion of main flow

- - Fuel jet boundary

(a) Case-22.5

(b) Case- 45

(b) Case- 45
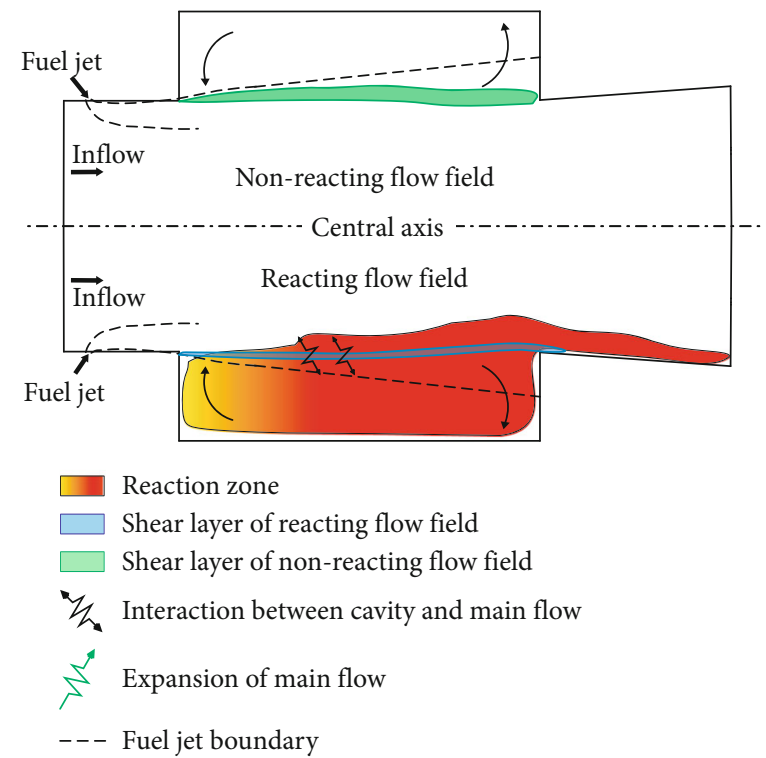

(c) Case- 90

(c) Case-90

FIgURE 16: Schematic of fuel mixing and combustion characteristics for all cases.

could be better to enlarge the aft wall angle properly when designing the cavity-assisted scramjet. Another interesting phenomenon is that the current simulation indicates that there exist some similarities between the rectangular and axisymmetric scramjets.

\section{Conclusions}

Numerical investigation is performed to explore the characteristics of the flow field and the effect of the cavity aft wall angle in an axisymmetric model scramjet. The simulation results are verified by the experimental data, and the mesh independency is tested. The fuel mixing and combustion characteristics of the configuration with the cavity aft wall angle of $22.5^{\circ}$ are analyzed in details. And then, two more configurations with cavity aft wall angles of $45^{\circ}$ and $90^{\circ}$ are compared to optimize the configuration of the scramjet. The main conclusions are as follows:

(1) The shear layer of the current axisymmetric cavity shows some similarities to that of the rectangular cavity in the nonreacting flow field. Both of them bend to the cavity floor in the middle of the cavity. The relatively larger volume of the cavity of axisymmetric scramjet leads to violent reaction and heat release of the combustion. The flow field, especially the cavity 
shear layer, is changed hugely by combustion in the axisymmetric scramjet. The combustion in the reacting flow field also leads to the nonaxisymmetric fuel mass exchange near the cavity shear layer

(2) In the nonreacting flow field, the thickness of the cavity shear layer decreases and the cavity shear layer becomes straight because of the reduced expansion of the main flow, when the aft wall angle increases. More fuel can be entrained and mix with the air of the supersonic inflow. The fuel distributes more uniformly around the cavity and the fuel mixing efficiency improves when the aft wall angle increases. It is beneficial to the fuel mixing performance of the axisymmetric scramjet to enlarge the aft wall angle of cavity properly

(3) In the reacting flow field, when the cavity aft wall angle increases, the interaction between cavity and main flow decreases, but the size of the hightemperature area inside the cavity increases. The former leads to poor fuel mixing and combustion performance, while the latter results in strong flameholding effect of the cavity. The combustion efficiency shows that the combustion performance improves when the aft wall angle increases. The combustion performance improves with the increasing of the cavity aft wall angle

(4) For the current axisymmetric cavity-assisted scramjet, a large cavity aft wall angle could improve the performance of the fuel mixing and combustion. It is recommended to increase the aft wall angle properly to achieve ideal performance when designing the cavity flameholder of scramjet

\section{Data Availability}

The data used to support the findings of this study are included within the article.

\section{Conflicts of Interest}

The authors declare that there is no conflict of interests regarding the publication of this paper.

\section{Acknowledgments}

This work is supported by the National Natural Science Foundation of China (Grant Nos. 11925207 and 12002381), the Scientific Research Plan of National University of Defense Technology in 2019 (Grant No. ZK19-02), the Postgraduate Scientific Research Innovation Project of Hunan Province (Grant No. CX20200084), and the Science and Technology Foundation of State Key Laboratory (Grant No. 6142703200311).

\section{References}

[1] G. Choubey, Y. D, W. Huang, L. Yan, H. Babazadeh, and K. M. Pandey, "Hydrogen fuel in scramjet engines - a brief review,"
International Journal of Hydrogen Energy, vol. 45, no. 33, pp. 16799-16815, 2020.

[2] J. F. de Araujo Martos, I. da Silveira Rêgo, S. N. Pachon Laiton, B. C. Lima, F. J. Costa, and P. G. de Paula Toro, "Experimental investigation of Brazilian 14-X B hypersonic scramjet aerospace vehicle," International Journal of Aerospace Engineering, vol. 2017, Article ID 5496527, 10 pages, 2017.

[3] M. Bulman and A. Siebenhaar, "The rebirth of round hypersonic propulsion," in 42nd AIAA/ASME/SAE/ASEE Joint Propulsion Conference \& Exhibit, Sacramento, CA, USA, July 2006.

[4] Y. Sun, M. Sun, J. Zhu et al., "Plif measurements of instantaneous flame structures and curvature of an acoustically excited turbulent premixed flame," Aerospace Science and Technology, vol. 104, article 105950, 2020.

[5] D. M. Peterson, R. R. Boyce, and V. Wheatley, "Simulations of mixing in an inlet-fueled axisymmetric scramjet," AIAA Journal, vol. 51, no. 12, pp. 2823-2832, 2013.

[6] W. O. Landsberg, N. N. Gibbons, V. Wheatley, M. K. Smart, and A. Veeraragavan, "Improving scramjet performance through flow field manipulation," Journal of Propulsion and Power, vol. 34, no. 3, pp. 578-590, 2018.

[7] Q. Liu, D. Baccarella, B. McGann, T. Lee, and H. Do, "Experimental investigation of single jet and dual jet injection in a supersonic combustor," in 2018 AIAA Aerospace Sciences Meeting, Kissimmee, FL, USA, January 2018.

[8] Y. Tian, S. Yang, and J. Le, "Study on the effect of air throttling on flame stabilization of an ethylene fueled scramjet combustor," International Journal of Aerospace Engineering, vol. 2015, 10 pages, 2015.

[9] G. Choubey and K. M. Pandey, "Numerical studies on the performance of scramjet combustor with alternating wedgeshaped strut injector," International Journal of Turbo \& JetEngines, vol. 34, no. 1, 2017.

[10] J. Yang, X. Wu, and Z. Wang, "Parametric study of fuel distribution effects on a kerosene-based scramjet combustor," International Journal of Aerospace Engineering, vol. 2016, Article ID 7604279, 14 pages, 2016.

[11] Z. Li, R. Moradi, S. M. Marashi, H. Babazadeh, and G. Choubey, "Influence of backward-facing step on the mixing efficiency of multi microjets at supersonic flow," Acta Astronautica, vol. 175, pp. 37-44, 2020.

[12] S. Aradag, K. A. Gelisli, and E. C. Yaldir, "Effects of active and passive control techniques on Mach 1.5 cavity flow dynamics," International Journal of Aerospace Engineering, vol. 2017, Article ID 8253264, 24 pages, 2017.

[13] T. Vanyai, S. Grieve, O. Street et al., "Fundamental scramjet combustion experiments using hydrocarbon fuel," Journal of Propulsion and Power, vol. 35, no. 5, pp. 953-963, 2019.

[14] Z. J. Denman, W. Y. K. Chan, S. Brieschenk, A. Veeraragavan, V. Wheatley, and M. K. Smart, "Ignition experiments of hydrocarbons in a Mach 8 shape-transitioning scramjet engine," Journal of Propulsion and Power, vol. 32, no. 6, pp. 1462-1471, 2016.

[15] Q. Liu, D. Baccarella, and T. Lee, "Influences of cavity on combustion stabilization in an axisymmetric scramjet," in AIAA SciTech 2019 Forum, San Diego, CA, USA, January 2019.

[16] F. Li, M. Sun, Z. Cai et al., "Experimental study of flame stabilization in a single-side expansion scramjet combustor with different cavity length-to-depth ratios," Acta Astronautica, vol. 173, pp. 1-8, 2020. 
[17] G. Choubey and K. M. Pandey, "Effect of different wall injection schemes on the flow-field of hydrogen fuelled strutbased scramjet combustor," Acta Astronautica, vol. 145, pp. 93-104, 2018.

[18] Y. Yang, Z. Wang, M. Sun, H. Wang, and L. Li, "Numerical and experimental study on flame structure characteristics in a supersonic combustor with dual-cavity," Acta Astronautica, vol. 117, pp. 376-389, 2015.

[19] X. Zhang, A. Rona, and A. Edwards, "The effect of trailing edge geometry on cavity flow oscillation driven by a supersonic shear layer," Aeronautical Journal, vol. 102, pp. 129-136, 1998.

[20] S. Jeyakumar, S. M. Assis, and K. Jayaraman, "Effect of axisymmetric aft wall angle cavity in supersonic flow field," International Journal of Turbo \& Jet-Engines, vol. 35, no. 1, pp. 2934, 2018.

[21] W. Yao, Y. Yuan, X. Li, J. Wang, K. Wu, and X. Fan, "Comparative study of elliptic and round scramjet combustors fueled by Rp-3," Journal of Propulsion and Power, vol. 34, no. 3, pp. 772786, 2018.

[22] Q. Liu, D. Baccarella, B. McGann, and T. Lee, "Cavityenhanced combustion stability in an axisymmetric scramjet model," AIAA Journal, vol. 57, no. 9, pp. 3898-3909, 2019.

[23] Q. Liu, D. Baccarella, W. Landsberg, A. Veeraragavan, and T. Lee, "Cavity flameholding in an optical axisymmetric scramjet in Mach 4.5 flows," Proceedings of the Combustion Institute, vol. 37, no. 3, pp. 3733-3740, 2019.

[24] G. Ma, M. Sun, F. Li, Y. Yang, Y. Huang, and H. Wang, "Effect of fuel injection distance and cavity depth on the mixing and combustion characteristics of a scramjet combustor with a rear-wall-expansion cavity," Acta Astronautica, vol. 182, pp. 432-445, 2021.

[25] A. Saghafian, V. E. Terrapon, and H. Pitsch, "An efficient flamelet-based combustion model for compressible flows," Combustion and Flame, vol. 162, no. 3, pp. 652-667, 2015.

[26] M. Zhao and T. Ye, "Urans study of pulsed hydrogen jet characteristics and mixing enhancement in supersonic crossflow," International Journal of Hydrogen Energy, vol. 44, no. 36, pp. 20493-20503, 2019.

[27] L. Li, W. Huang, L. Yan, Z. Zhao, and L. Liao, "Mixing enhancement and penetration improvement induced by pulsed gaseous jet and a vortex generator in supersonic flows," International Journal of Hydrogen Energy, vol. 42, no. 30, pp. 19318-19330, 2017.

[28] G. Zhao, M. Sun, H. Wang, and H. Ouyang, "Investigation of combustion characteristics in a scramjet combustor using a modified flamelet model," Acta Astronautica, vol. 148, pp. 32-40, 2018.

[29] G. Zhao, M. Sun, J. Wu, and H. Wang, "A flamelet model for supersonic non-premixed combustion with pressure variation," Modern Physics Letters B, vol. 29, no. 21, article 1550117, 2015.

[30] Y. Sun, M. Sun, J. Zhu et al., "The local extinction and the nonlinear behaviors of a premixed methane/air flame under lowfrequency acoustic excitation," Modern Physics Letters B, vol. 34, no. 13, article 2050138, 2020.

[31] M. V. Petrova, Detailed and Reduced Chemical-Kinetic Descriptions for Hydrocarbon Combustion, University of California, San Diego, CA, USA, 2005.

[32] A. L. Sánchez and F. A. Williams, "Recent advances in understanding of flammability characteristics of hydrogen," Progress in Energy and Combustion Science, vol. 41, pp. 1-55, 2014.
[33] H. Pitsch, Flamemaster: A C++ Computer Program for Od Combustion and 1d Laminar Flame Calculations, 1998, http://www.itv.rwth-aachen.de/downloads/flamemaster.

[34] W. Huang, J. Liu, L. Jin, and L. Yan, "Molecular weight and injector configuration effects on the transverse injection flow field properties in supersonic flows," Aerospace Science and Technology, vol. 32, no. 1, pp. 94-102, 2014.

[35] T. Gao, J. Liang, M. Sun, and Z. Zhong, "Investigation of asymmetric and unsteady combustion in a supersonic combustor with single-side expansion," Journal of Propulsion Technology, vol. 37, pp. 419-427, 2016.

[36] H. Wang, Z. Wang, M. Sun, and N. Qin, "Experimental and numerical investigation of cavity-based supersonic flow and combustion," Proceedings of the Institution of Mechanical Engineers, Part G: Journal of Aerospace Engineering, vol. 228, pp. 781-798, 2013.

[37] F. Li, M. Sun, Z. Cai et al., "Effects of additional cavity floor injection on the ignition and combustion processes in a Mach 2 supersonic flow," Energies, vol. 13, no. 18, article 4801, 2020.

[38] M. Sun and Z. Hu, "Formation of surface trailing counterrotating vortex pairs downstream of a sonic jet in a supersonic cross-flow," Journal of Fluid Mechanics, vol. 850, pp. 551-583, 2018.

[39] Z. Du, W. Huang, L. Yan, L. Li, Z. Chen, and S. Li, "Rans study of steady and pulsed gaseous jets into a supersonic crossflow," International Journal of Heat and Mass Transfer, vol. 136, pp. 157-169, 2019.

[40] H. Wang, Z. Wang, M. Sun, and H. Wu, "Combustion modes of hydrogen jet combustion in a cavity-based supersonic combustor," International Journal of Hydrogen Energy, vol. 38, no. 27, pp. 12078-12089, 2013. 